\title{
Synthesis of High Magnetization Fe and FeCo nanoparticles by High Temperature Chemical Reduction
}

\author{
Binil Kandapallil, ${ }^{\text {a }}$ Robert E. Colborn, Peter J. Bonitatibus, and Frank Johnson \\ General Electric Global Research Centre, 1 Research Circle, Niskayuna, NY 12309
}

\begin{abstract}
Fe and FeCo ferromagnetic nanoparticles in the 5-10 $\mathrm{nm}$ size regimes featuring high magnetization were synthesized using a modified chemical reduction method. The structure and morphology of these nanoparticles were confirmed by XRD and TEM analysis. These small, monodisperse and phase pure nanoparticles exhibited magnetic saturation of $210 \mathrm{emu} / \mathrm{g}(\mathrm{Fe})$ and $220 \mathrm{emu} / \mathrm{g}(\mathrm{Fe}+\mathrm{Co})$ for $\mathrm{Fe}$ and FeCo nanoparticles respectively. The magnetization was found to be dependent on the temperature at which the reducing agent was introduced.

Keywords: Nanoparticles, Ferromagnetic, Iron, Iron-Cobalt, Saturation Magnetization, Chemical reduction

\section{INTRODUCTION}

The synthesis of monodisperse soft ferromagnetic nanoparticles of Fe and FeCo possessing excellent magnetic properties has attracted significant interest in recent years. ${ }^{1-5}$ There is an ever increasing demand to achieve quality soft-phase nanoparticles because of their potential applications in magnetic storage, ${ }^{6}$ high performance inductors, ${ }^{7}$ biomedicine ${ }^{8}$ and nanocomposite permanent magnets. ${ }^{9}$ Many methods have been developed for their synthesis which includes chemical (polyol, ${ }^{1}$ reduction of $\mathrm{Fe}^{2+}$ and $\mathrm{Co}^{2+}$ ions using reductants, ${ }^{10,11}$ decomposition from carbonyls ${ }^{4}$ ) as well as mechanical, ${ }^{12}$ electrochemical, ${ }^{13}$ and chemical vapor deposition techniques. ${ }^{14}$ However there is an overarching goal for developing a scalable process which enables the synthesis of small (5-10 nm), phase pure (absence of oxides and nonmagnetic byproducts), monodisperse nanoparticles possessing high magnetic saturation values. This small size requirement for ferromagnetic nanoparticles may be important to achieve efficient interparticle magnetic interactions like the spring-coupling in a nanocomposite permanent magnet. ${ }^{15}$
\end{abstract}

\footnotetext{
${ }^{a}$ Author to whom correspondence should be addressed. Electronic mail:kandapal@ge.com
} 
In this study, we present an efficient and inexpensive scalable synthetic route to achieve Fe and FeCo nanoparticles in the 5-10 nm size regimes, by chemically reducing the precursor metal salts in a high boiling solvent under an inert atmosphere. The resulting $\mathrm{Fe}(0)$ nanoparticles exhibited magnetic saturation values of $210 \mathrm{emu} / \mathrm{g}(\mathrm{Fe})$, identical to bulk theoretical values while FeCo(0) nanoparticles exhibited an $\mathrm{M}_{\text {sat }}$ of 220 emu/g(Fe $\left.+\mathrm{Co}\right)$, after annealing the sample at $500{ }^{\circ} \mathrm{C}$. The relevance of reduction temperature on the magnetic behavior of these ferromagnetic nanoparticles has also been elucidated in this work.

\section{EXPERIMENTAL PROCEDURES}

In a typical experimental procedure for Fe nanoparticle synthesis, a 3-necked round bottom flask fitted with an air condenser and thermocouple was loaded with $1.5 \mathrm{~g} \mathrm{FeBr}_{2}(7$ mmol). The reaction flask was kept under an argon atmosphere, wherein diphenyl ether (40 $\mathrm{mL}$ ) was added using a syringe followed by addition of oleic acid $(0.7 \mathrm{~mL}, 2.2 \mathrm{mmol})$. This mixture was heated to $100{ }^{\circ} \mathrm{C}$, and incubated under vacuum for 1 hour for degassing. The reaction was switched back to inert atmosphere and trioctylphosphine $(2.7 \mathrm{~mL}, 6 \mathrm{mmol})$ was injected to the degassed mixture. The reaction mixture was heated to $290{ }^{\circ} \mathrm{C}$ and after attaining the temperature, lithium triethylborohydride $(14 \mathrm{~mL}$ of $1 \mathrm{M}$ solution in THF, $14 \mathrm{mmol})$ was injected drop-wise. Under these conditions, THF was removed by condensation in a cooled receiver connected to the condenser. This reaction was maintained at $290{ }^{\circ} \mathrm{C}$ for 3 hours followed by cooling to room temperature. Although the boiling point of pure solvent is $265^{\circ} \mathrm{C}$, it may be noted here that the presence of large quantities of non-volatile metal salt precursor enables reaching higher temperatures up to $290{ }^{\circ} \mathrm{C}$ utilizing the colligative property.

For the FeCo nanoparticle synthesis, a very similar process was adopted. A 3-necked round bottom flask fitted with an air condenser and thermocouple was loaded with $1.5 \mathrm{~g} \mathrm{FeBr}_{2}$ (7 mmol), $0.654 \mathrm{~g}$ and $\mathrm{CoBr}_{2}(3 \mathrm{mmol})$. To this reaction flask kept under an argon atmosphere, diphenyl ether $(40 \mathrm{~mL})$ was syringed in followed by oleic acid $(0.7 \mathrm{~mL}, 2.2 \mathrm{mmol})$. The mixture

was heated to $100{ }^{\circ} \mathrm{C}$, and left under vacuum for 1 hour. The reaction was switched back to inert atmosphere and trioctylphosphine $(4 \mathrm{~mL}, 10 \mathrm{mmol})$ was injected. The reaction was heated to $290{ }^{\circ} \mathrm{C}$ and after attaining the temperature, lithium triethylborohydride $(20 \mathrm{~mL}$ of $1 \mathrm{M}$ 
solution in THF, $20 \mathrm{mmol}$ ) was injected drop-wise. Under these conditions, THF was removed by condensation in a cooled receiver connected to the condenser. The reaction was maintained at $290{ }^{\circ} \mathrm{C}$ for 3 hours followed by cooling to room temperature.

In both cases, the reaction mixture under inert atmosphere was carefully transferred into a glovebox where the product was precipitated and washed multiple times before characterization. The nanoparticles inside the glovebox were precipitated by adding a polar protic anti-solvent such as ethanol (5:1 of ethanol: hexane) into the reaction mixture. The precipitated nanoparticles were separated and retained using a permanent magnet. The nanoparticles were re-suspended in hexanes and further precipitated using ethanol. The process was continued one more time and the nanoparticles were dried under vacuum by connection to a vacuum pump attached to the glovebox.

Heat treatment was done in a quartz tube under an argon atmosphere. The sample was transferred into a quartz tube fitted with a gas regulator inside a glovebox. The closed sample tube was transferred into the heat treatment oven and connected to a supply of argon gas. All further steps were carried out under a continuous flow of argon. The temperature ramp was carried out as follows: (i) from room temperature to $300^{\circ} \mathrm{C}, 10^{\circ} \mathrm{C} / \mathrm{min}$; (ii) from $300^{\circ} \mathrm{C}$ to $500^{\circ} \mathrm{C}$, $5^{\circ} \mathrm{C} / \mathrm{min}$; (iii) $30 \mathrm{~min}$ at $500^{\circ} \mathrm{C}$, then natural cooling back to room temperature. The sample was isolated using the regulator before transferring into the glovebox.

The purified Fe nanoparticles were transferred to a plastic sample holder inside a glovebox. The magnetic hysteresis was performed on a Physical Property Measurement System (PPMS) from Quantum Design. The measurement was carried out at room temperature with a field sweep of \pm 7 tesla. The system was equipped with a sealed sample chamber. XRD was performed on an EQUINOX 5000 Inel machine (50 KV*80 mA) fitted with Rigaku rotating anode X-ray generator and Inel curved 1-D position sensitive detector. Data was generated using Mo $\mathrm{K}$ radiation in the transmission mode and later converted to $\mathrm{Cu} \mathrm{K}$ radiation peaks. The sample was mounted on a sealed spinning glass capillary inside the glovebox prior to measurements. The TEM (transmission electron microscopy) image of the nanoparticles was analyzed using a FEI Tecnai 200kV system fitted with a Thermo Scientific EDS (energy dispersive spectroscopy) system. 


\section{RESULTS AND DISCUSSION}

The TEM image and EDS pattern of the Fe nanoparticles synthesized are represented in

Fig. 1. The mean diameter of the iron nanoparticles is about $5 \mathrm{~nm}$, as reflected from the inset

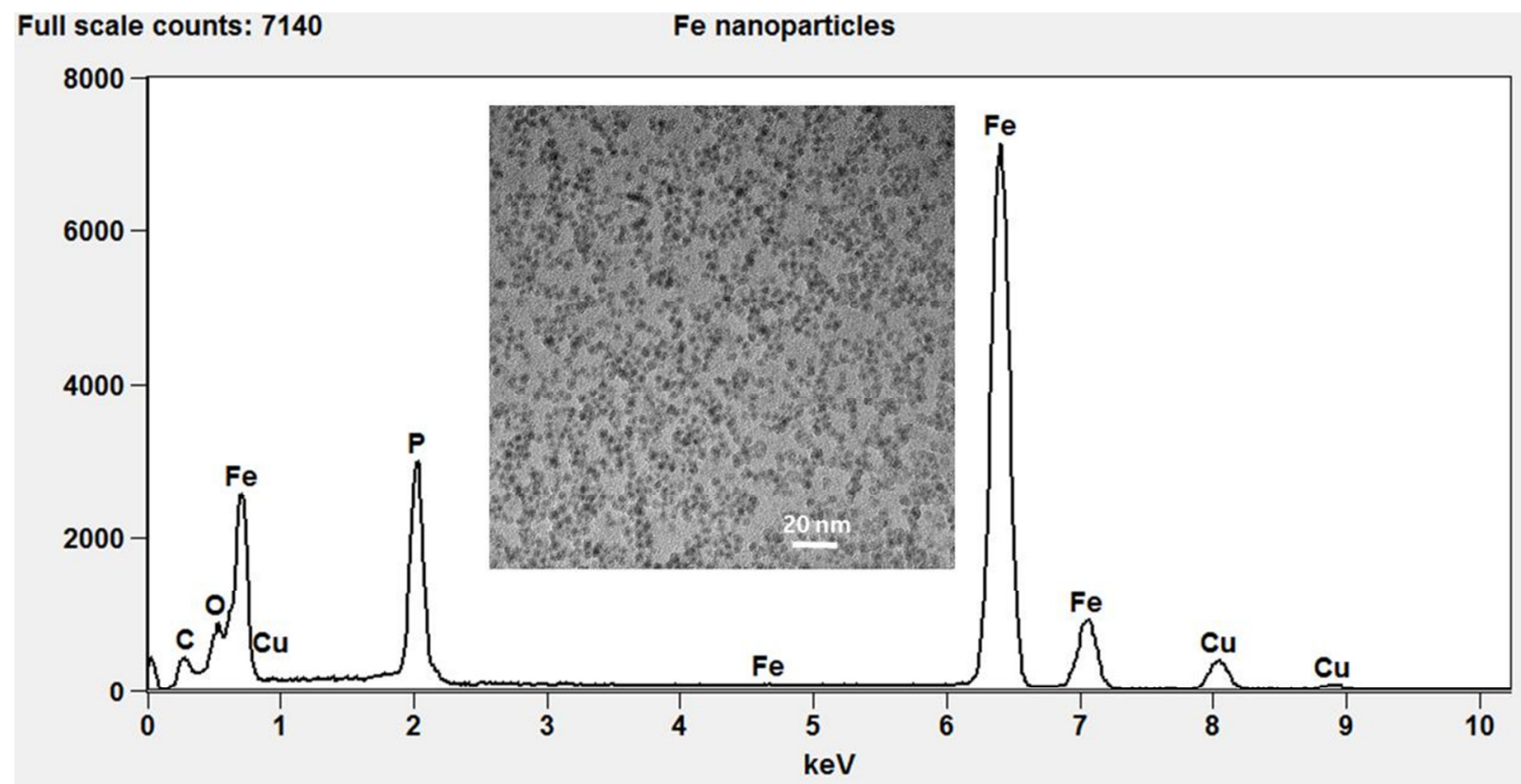

Figure 1. EDS of as synthesized Fe nanoparticles. Inset shows the TEM image of the corresponding nanoparticles. 


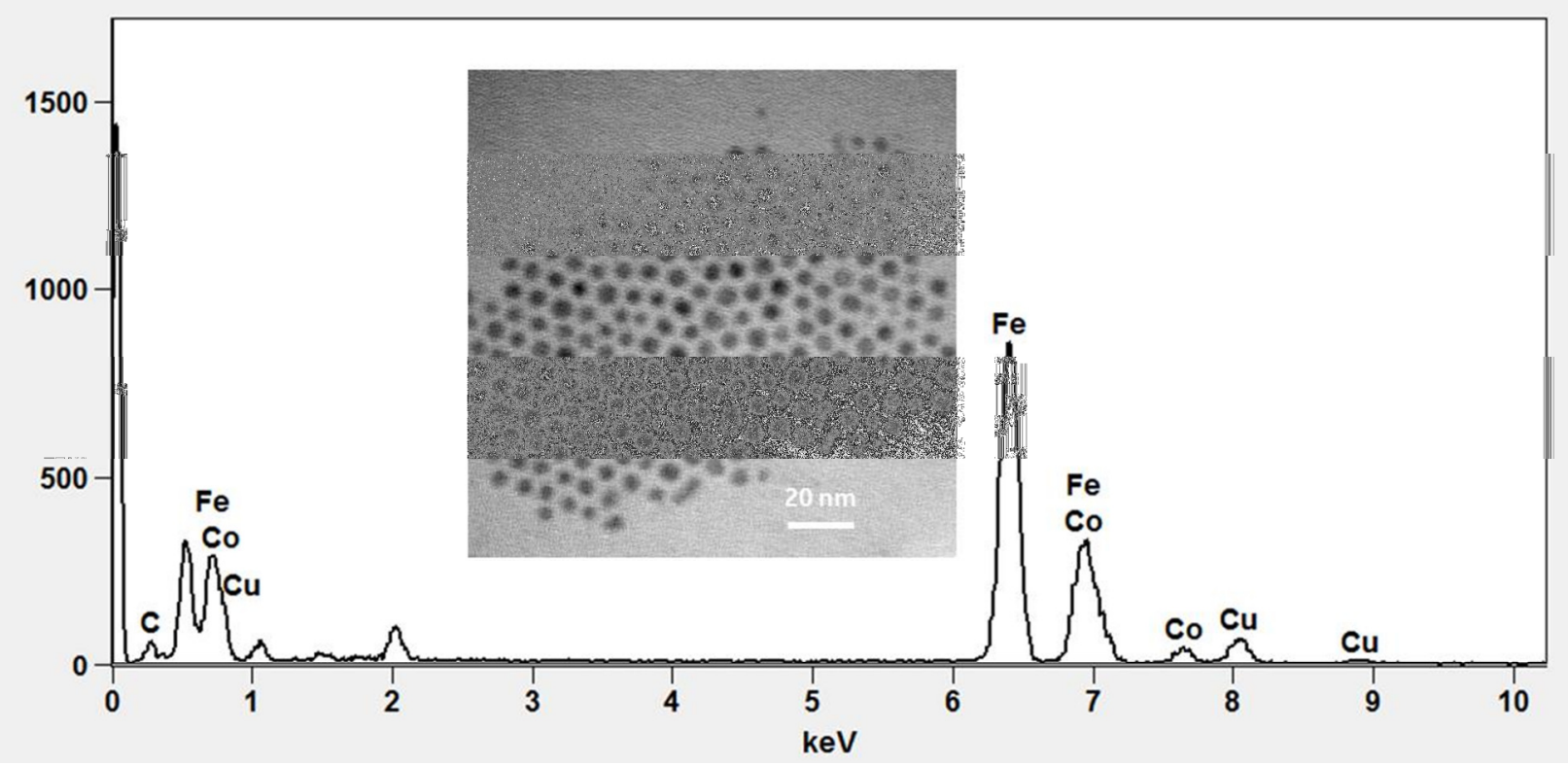

Figure 2. EDS of as synthesized FeCo nanoparticles. Inset shows the TEM image of the corresponding nanoparticles.

of Fig. 1. While the Fe nanoparticles are near spherical, the FeCo nanoparticles are more spherical in nature. Although the sample preparation for TEM is carried out inside a glovebox, the samples get transiently exposed to air during sample mounting into the microscope. Therefore, a thin oxide-layer on these nanoparticles in unavoidable. The EDS peaks correspond to Fe nanoparticles and the background peaks correspond to the ligand chemicals present on the nanoparticle surface. The size of the iron-cobalt (FeCo) nanoparticles is $<5 \mathrm{~nm}$, as illustrated in Fig. 2. The EDS pattern of the FeCo nanoparticles synthesized is also represented in Fig. 2, wherein the EDS peaks correspond to FeCo nanoparticles and the background peaks correspond to the ligand chemicals present in the coating of the nanoparticles. The ratio of Fe and $\mathrm{Co}$ peaks from the EDS also corresponds to a mole ratio of $\mathrm{Fe}_{66} \mathrm{CO}_{34}$, consistent with the amount of material loaded into the reactor. It can be noted that there are no peaks corresponding to Fe or Co nanoparticles in the EDS spectrum. The crystallinity and monodisperse nature of these as synthesized nanoparticles is clearly manifested from their TEM images. 


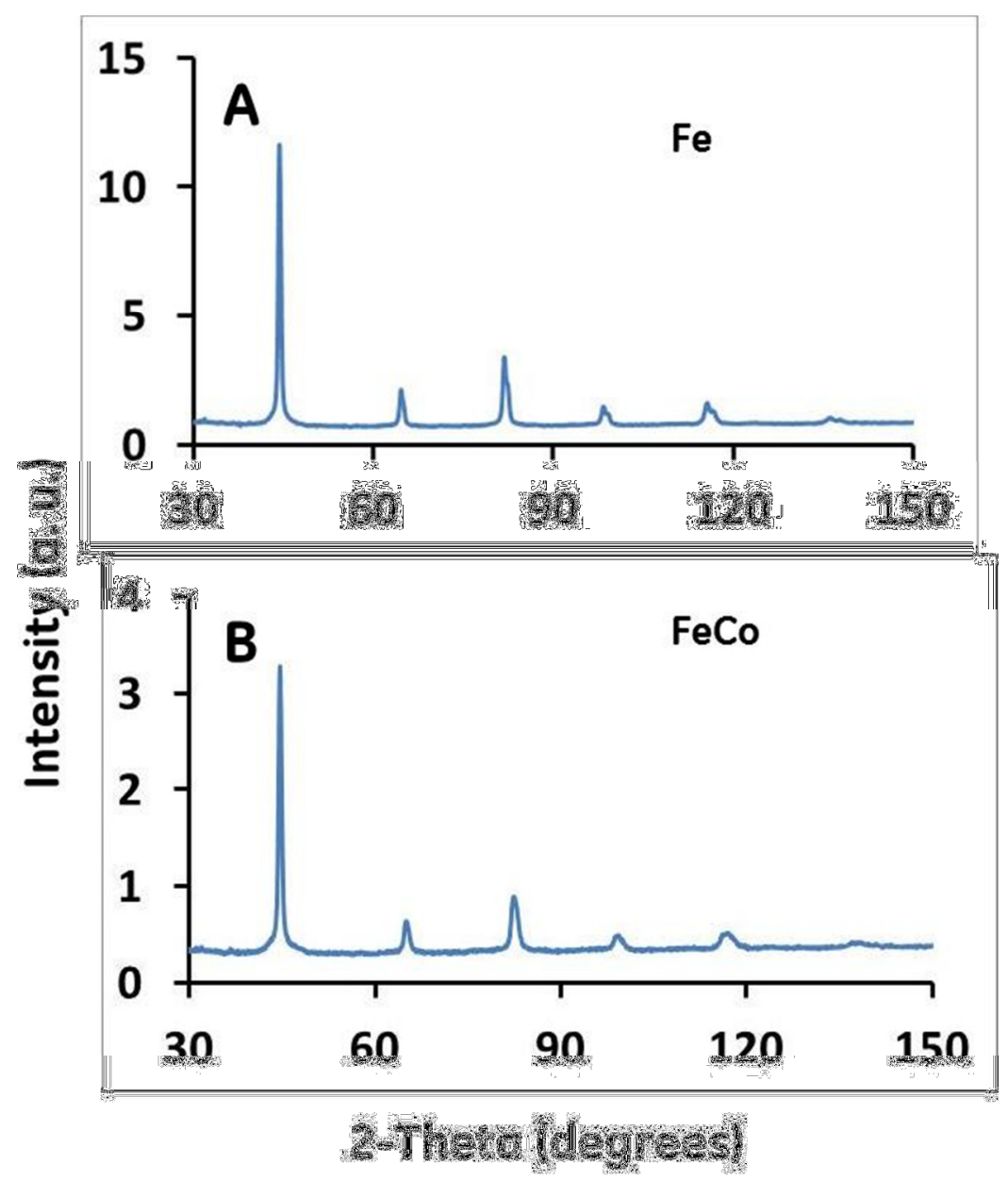

Figure 3. XRD pattern of as synthesized nanoparticles of A) Fe and B) FeCo.

Fig. $3 \mathrm{~A}$ and $3 \mathrm{~B}$ shows the powder X-ray diffraction (XRD) pattern from as synthesized Fe and FeCo nanoparticles respectively. The diffraction peaks can be assigned to the (110), (200) and (211) reflections of the bcc Fe and FeCo alloy nanoparticles. The sharp XRD peaks indicate the crystalline nature of these nanoparticles. The purified nanoparticles inside a glovebox are typically transferred into capillary tubes for XRD characterization, leaving no opportunity for airoxidation. These nanoparticles were thoroughly cleaned by stripping the ligands using ethanol washing and magnetic separation (experimental methods), which renders cleaner and narrower Bragg diffraction from these crystallites (Figure 3). Moreover, the larger nanoparticles in the ensemble might have contributed to the overall peak structure in the diffraction pattern. 


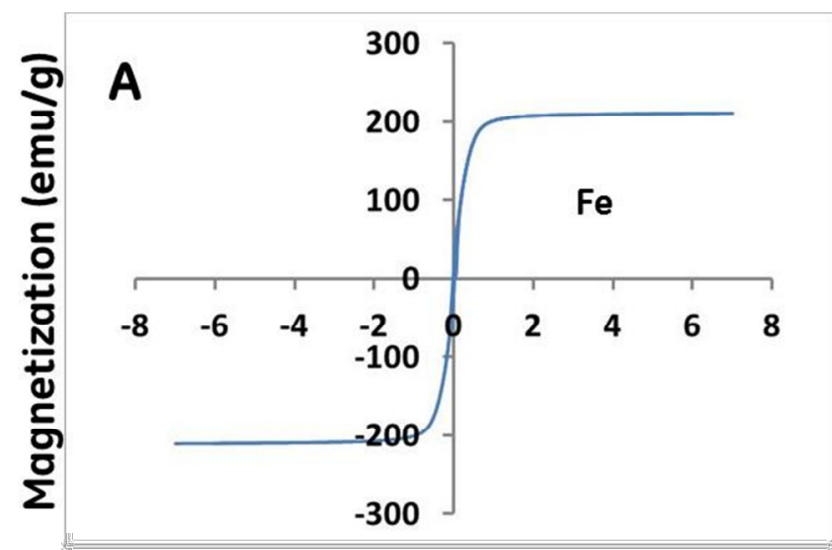

Applied Field (KOe)

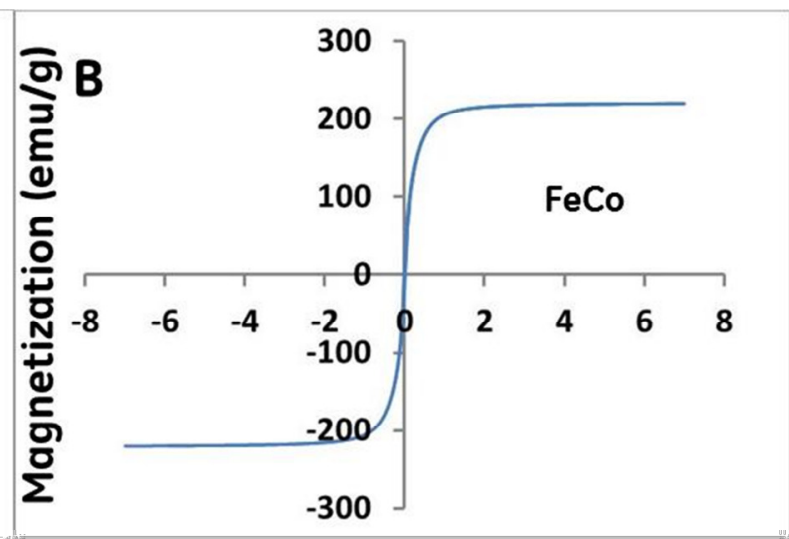

Applied field (Koe)

Figure 4. Hysteresis loop of A) Fe and B) FeCo nanoparticles.

In order to quantitatively measure the amount of Fe and FeCo in each sample for Msat measurement, elemental analysis was performed on these samples after washing with ethanol and subsequent annealing process. Fig. 4A, B represents the room temperature hysteresis loop from annealed samples of $\mathrm{Fe}$ and FeCo nanoparticles respectively. The saturation magnetizations (Msat) of 210 and $220 \mathrm{emu} / \mathrm{g}(\mathrm{Fe}+\mathrm{Co})$ were obtained for Fe and FeCo nanoparticles. It may also be noted that these nanoparticles did not exhibit any coercivity at room temperature. We did a systematic study of the effect of reduction temperature on the Msat characteristics of resulting Fe nanoparticles. The reaction mixtures for three different sets were heated to $200^{\circ} \mathrm{C}, 250^{\circ} \mathrm{C}$ and $290^{\circ} \mathrm{C}$ and after attaining the temperature; lithium triethylborohydride was injected drop wise as the reducing agent. The reaction was then maintained at $200^{\circ} \mathrm{C}, 250^{\circ} \mathrm{C}$ and $290^{\circ} \mathrm{C}$ for 3 hours followed by cooling to room temperature. The corresponding Msat values were measured and are summarized in Table 1. It is obvious that higher temperature results in higher saturation values presumably due to the formation of nanoparticles with higher crystallinity. Msat value of $210 \mathrm{emu} / \mathrm{g}(\mathrm{Fe})$ attained here is close to the bulk theoretical value indicating that annealing is not always necessary to produce high quality nanoparticles. 
Table 1. Temperature dependence of reduction on Msat of Fe nanoparticles

\begin{tabular}{|c|c|}
\hline $\begin{array}{l}\text { Reduction } \\
\text { temperature }\left({ }^{\circ} \mathbf{C}\right)\end{array}$ & $\mathbf{M}_{\text {sat }}$ (emu/g(Fe) \\
\hline 200 & 188 \\
\hline 250 & 200 \\
\hline 290 & 210 \\
\hline
\end{tabular}

In the case of FeCo nanoparticles, annealing was necessary to attain higher values of saturation magnetization indicating that inter-diffusion of Co into the Fe lattice is a higher energy process. Interestingly after the annealing step, there is no noticeable change in the size of these nanoparticles (Figure 5). The background that is seen in the TEM image may be the formation of carbonaceous material during the annealing process. In general, it may be noted that sample handling is performed under strict air-free conditions, which might have contributed to the production of high quality magnetic nanoparticles. The synthesis was also carried out at $20 \mathrm{~g}$ scales resulting in high quality nanoparticles of both Fe and FeCo, characterized by their small size and superior magnetic properties.

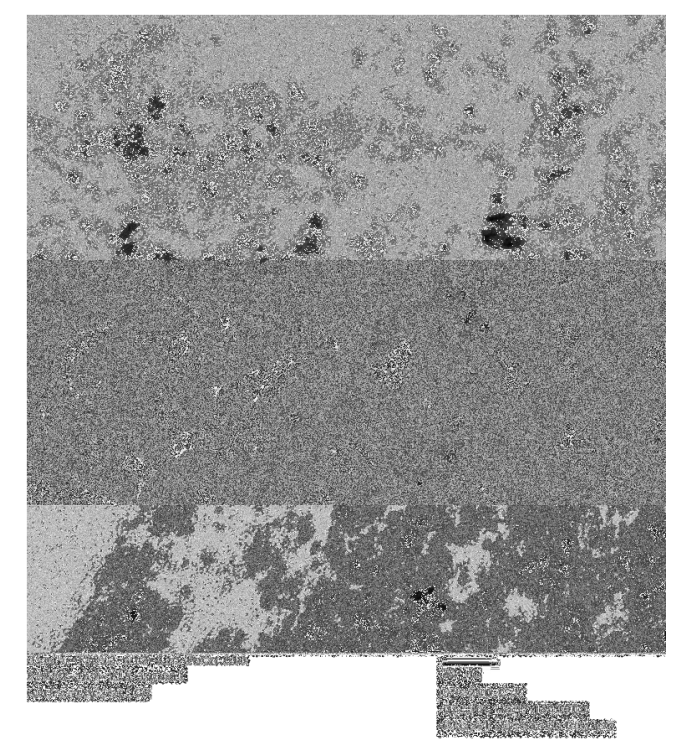

Figure 5. TEM image of FeCo nanoparticles after annealing. 


\section{CONCLUSIONS}

Small $(5-10 \mathrm{~nm})$ Fe and FeCo nanoparticles with high quality magnetic properties were synthesized by a chemical reduction process. A systematic study on the reduction temperature of metal ions was studied and has been optimized to attain high magnetic properties. The Magnetic saturation values were 210 and $220 \mathrm{emu} / \mathrm{g}(\mathrm{Fe}+\mathrm{Co})$ for Fe, FeCo nanoparticles respectively, almost identical to bulk values. TEM, XRD and EDS have been used to characterize these nanoparticles and they exhibit excellent crystallinity and phase purity. High temperature annealing was performed on FeCo nanoparticles to improve their crystallinity without impacting their size characteristics. These small, highly magnetic nanoparticles could have potential applications in engineering applications like nanocomposite permanent magnets. ${ }^{16}$

\section{ACKNOWLEDGEMENT}

This information data, or work presented herein was funded in part by the Advanced Research Projects Agency- Energy (ARPA-E) U.S. Department of Energy under Award Number DE-AR0000158. We acknowledge the help from Orrie Riccobono, Jay Hyuk Her, Chris Dosch for TEM, EDS and XRD characterization of the nanoparticle samples. 


\section{References}

1. Z. J. Huba, K. J. Carroll, and E. E. Carpenter, J. Appl. Phys. 109, 07B514 (2011)

2. V. Tzitzios, G. Basina, D. Niarchos, W. Li, and G. Hadjipanayis, J. Appl. Phys. 109, 07 A313 (2011)

3. G. S. Chaubey, C. Barcena, N. Poudyal, C. Rong, J. Gao, S. Sun, and J. P. Liu, J. Am. Chem. Soc. 129, 7214 (2007)

4. C. Desvaux, F. Dumestre, C. Amiens, M. Respaud, P. Lecante, E. Snoeck, P. Fejes, P. Renaud, and B. Chaudret, J. Mater. Chem. 19, 3268 (2009).

5. C. Desvaux, C. Amiens, P. Fejes, P. Renaud, M. Respaud, P. Lecante, E. Snoeck, and B. Chaudret, Nature Mater. 4, 750 (2005).

6. F. Jorgensen, The Complete Handbook of Magnetic Recording (McGraw-Hill, New York, 1995).

7. P. C. P. Watts, W. K. Hsu, D. P. Randall, V. Kotzeva, and G. Z. Chen, Chem. Mater. 14, 4505 (2002).

8. J. Bai and J. Wang, Appl. Phys. Lett. 87, 152502 (2005).

9. F. E. Kneller and R. Hawig, IEEE Trans, Magn. 27, 3588 (1991).

10. K. E. Mooney and M. J. Wagner, J. Mater. Chem. 19, 611(2009).

11. J. Kim, J. Kim, J. Kim, and K. H. Kim, J. Appl. Phys. 113, $17 A 313$ (2013).

12. L. Kerekes, J. Hakl, S. Meszaros, K. Vad, P. Gurin, I. Kis-Varga, I. Uzonyi, S. Szabo, and D. Beke, Czech. J. Phys. 52, A89-A92 (2002).

13. A. Bai and C. C. Hu, Electrochem. Commun. 5. 78 (2003).

14. D. M. Cox, D. J. Trevor, R. L. Whetten, E. A. Rohlfing, and A. Kaldor, Phys. Rev. B 32, 7290-7298 (1985).

15. H. Zeng, J. Li, J. P. Liu, Z. L. Wang, and S. Sun, Nature 420, 395 (2002).

16. Disclaimer: The information, data, or work presented herein was funded in part by an agency of the United States Government. Neither the United States Government nor any agency thereof, nor any of their employees, makes any warranty, express or implied, or assumes any legal liability or responsibility for the accuracy, completeness, or usefulness of any information, apparatus, product, or process disclosed, or 
represents that its use would not infringe privately owned rights. Reference herein to any specific commercial product, process, or service by trade name, trademark, manufacturer, or otherwise does not necessarily constitute or imply its endorsement, recommendation, or favoring by the United States Government or any agency thereof. The views and opinions of authors expressed herein do not necessarily state or reflect those of the United States Government or any agency thereof. 